\title{
LOCAL COMPACTNESS IN APPROACH SPACES I
}

\author{
R. LOWEN \\ Department of Mathematics \\ University of Antwerp, RUCA \\ Groenenborgerlaan 171, B-2020 Antwerp, Belgium \\ C. Verbeeck \\ Department of Mathematics and Computer Science \\ University of Antwerp, UIA \\ Universiteitsplein 1, B-2610 Wilrijk, Belgium
}

(Received August 14, 1996)

\begin{abstract}
This paper introduces some definitions of a concept of local compactness in approach spaces. The basic relationships between these concepts is studied, and measures of local compactness are defined.
\end{abstract}

KEY WORDS AND PHRASES: Approach space, measure of compactness, (basis-) local compactness, measure of (basis-) local compactness.

1991 AMS SUBJECT CLASSIFICATION CODES: 54A05; 54D45.

\section{INTRODUCTION}

The purpose of this paper is to introduce and study some reasonable definitions of a concept of local compactness in approach spaces. The search for the right notion - if such a uniquely determined generalization exists - is motivated, not only by the obvious fact that local compactness is an important and natural concept in topology and hence, as has been made clear by the development of the theory so far, will be equally important in approach spaces, but more specifically it is motivated by the search for a description of the exponential objects in $A P$. In [1] this problem was successfully solved in $P R A P$, the category of pre-approach spaces. As is well-known however the situation in TOP as compared to PRTOP, the category of pretopological spaces, is considerably more complicated, and the same pattern presents itself in the theory of approach spaces. The link between notions of local compactness and exponential object in $A P$ will be the topic of forthcoming work. Unrelated to this problem however, we found that there are a number of intuitively appealing concepts in $A P$ which have nice properties and which even allow for quantification in the way Kuratowski's and Hausdorff's measures of non-compactness quantify the topological notion of compactness. In this first part of our paper we concentrate on a basic study of local compactness concepts and their relationship. 


\section{PRELIMINARIES}

Given a set $X$ we denote its power set by $2^{X}$ and the set of its finite subsets by $2^{(X)}$. We recall those concepts and results from Lowen $[2,3]$ which we require in the sequel. A map

$$
\delta: X \times 2^{X} \rightarrow[0, \infty]
$$

is called a distance if it fulfils

(D1) $\forall A \in 2^{X}, \forall x \in X: x \in A \Rightarrow \delta(x, A)=0$.

(D2) $\forall x \in X: \delta(x, \emptyset)=\infty$.

(D3) $\forall A, B \in 2^{X}, \forall x \in X: \delta(x, A \cup B)=\delta(x, A) \wedge \delta(x, B)$.

(D4) $\forall A \in 2^{X}, \forall x \in X, \forall \varepsilon \in[0, \infty]: \delta(x, A) \leq \delta\left(x, A^{(\varepsilon)}\right)+\varepsilon$ where

$$
A^{(\varepsilon)}:=\{x \mid \delta(x, A) \leq \varepsilon\}
$$

If $\delta$ is a distance on $X$ and $A \subset X$, the function $\delta_{A}: X \rightarrow[0, \infty]$ is defined by $\delta_{A}(x):=\delta(x, A)$.

- A collection $(\mathcal{A}(x))_{x \in X}$ of ideals in $[0, \infty]^{X}$ is called an approach system if it fulfils

(A1) $\forall x \in X, \forall \varphi \in \mathcal{A}(x): \varphi(x)=0$.

(A2) $\forall x \in X, \forall \varphi \in[0, \infty]^{X}$ :

$$
(\forall \varepsilon, N \in] 0, \infty\left[, \exists \varphi_{\varepsilon}^{N} \in \mathcal{A}(x): \varphi \wedge N \leq \varphi_{\varepsilon}^{N}+\varepsilon\right) \Rightarrow \varphi \in \mathcal{A}(x) .
$$

(A3) $\forall x \in X, \forall \varphi \in \mathcal{A}(x), \forall N \in] 0, \infty[$,

$$
\exists\left(\varphi_{z}\right)_{z \in X} \in \prod_{z \in X} \mathcal{A}(z), \forall z, y \in X: \varphi(y) \wedge N \leq \varphi_{x}(z)+\varphi_{z}(y) .
$$

The elements of an approach system are called local distances. For ease in notation we shall, whenever convenient denote an approach system $(\mathcal{A}(x))_{x \in X}$ also simply $\mathcal{A}$.

If $\mathcal{A}$ is an approach system then $\Lambda:=(\Lambda(x))_{x \in X}$ is called a basis for $\mathrm{A}$ if it fulfils the properties

(b1) $\forall x \in X: \Lambda(x)$ is a basis for an ideal.

(b2) $\forall x \in X: \mathcal{A}(x)=\hat{\Lambda}(x)$ where

$$
\hat{\Lambda}(x):=\left\{\varphi \in[0, \infty]^{X} \mid \forall \varepsilon, N \in\right] 0, \infty[, \exists \psi \in \Lambda(x): \varphi \wedge N \leq \psi+\varepsilon\} .
$$

\section{PROPOSITION 2.1 [3]}

1. If $\mathcal{A}$ is an approach system on $X$ then the map

$$
\delta_{\mathcal{A}}: X \times 2^{X} \rightarrow[0, \infty]:(x, A) \mapsto \sup _{\varphi \in \mathcal{A}(x)} \inf _{a \in A} \varphi(a)
$$

is a distance on $X$.

2. If $\delta$ is a distance on $X$ then the system $\mathcal{A}_{\delta}$ where for all $x \in X$

$$
\mathcal{A}_{\delta}(x):=\left\{\varphi \in[0, \infty]^{X} \mid \forall A \subset X: \inf _{a \in A} \varphi(a) \leq \delta(x, A)\right\}
$$

is an approach system on $X$. 
3. $\mathcal{A}_{\delta_{\mathcal{A}}}=\mathcal{A}$ and $\delta_{\mathcal{A}_{\delta}}=\delta$.

A set $X$ equipped with an approach system or equivalently a distance is called an approach space and is usually denoted $(X, \mathcal{A})$. The associated distance is usually denoted simply $\delta$ instead of $\delta_{\mathcal{A}}$ and analogously if $\delta$ is the primary defined structure, $\mathcal{A}_{\delta}$ is usually simply denoted $\mathcal{A}$, unless confusion might occur.

PROPOSITION 2.2 [3] If $\Lambda$ is a basis for $\mathcal{A}$ then $\delta_{\mathcal{A}}$ is also obtained by

$$
\delta_{\mathcal{A}}(x, A)=\sup _{\psi \in \Lambda(x)} \inf _{a \in A} \psi(a) .
$$

If $(X, \mathcal{A})$ and $\left(X^{\prime}, \mathcal{A}^{\prime}\right)$ are approach spaces and $f: X \rightarrow X^{\prime}$ is a map then $f$ is called a contraction if it fulfils any of the following equivalent (see Lowen [3]) conditions:

(c1) $\forall x \in X, \forall \varphi^{\prime} \in \mathcal{A}^{\prime}(f(x)): \varphi^{\prime} \circ f \in \mathcal{A}(x)$.

(c2) For any basis $\Lambda^{\prime}$ for $\mathcal{A}^{\prime}, \forall x \in X, \forall \psi^{\prime} \in \Lambda^{\prime}(f(x)): \psi^{\prime} \circ f \in \mathcal{A}(x)$.

(c3) $\forall x \in X, \forall A \subset X: \delta^{\prime}(f(x), f(A)) \leq \delta(x, A)$.

Approach spaces and contractions form a topological construct Lowen [3] which we denote $A P$. For categorical concepts, in particular topological categories, we refer to Adámek et al. [4]. TOP can be embedded as a bireflective and bicoreflective subconstruct of $A P$. The embeddingsfunctor is given by $(X, \mathcal{T}) \rightarrow\left(X, \mathcal{A}_{\mathcal{T}}\right)$ leaving morphisms unaltered and where $\mathcal{A}_{\mathcal{T}}$ is the approach system $\mathcal{A}_{\mathcal{T}}(x):=\left\{\varphi \in[0, \infty]^{X} \mid \varphi(x)=0, \varphi\right.$ u.s.c. at $\left.x\right\}$ for every $x \in X$, and which has as basis the collection $\left\{\theta_{V} \mid V\right.$ neighborhood of $x$ for $\left.\mathcal{T}\right\}$ for every $x \in X$, where $\theta_{V}(x)=0$ if $x \in V$ and $\theta_{V}(x)=\infty$ if $x \notin V$. The associated distance is given by $\delta(x, A)=0$ if $x \in \bar{A}$ and $\delta(x, A)=\infty$ if $x \notin \bar{A}$. The subconstruct thus obtained is isomorphic to TOP. We recall that the bicoreflection of $(X, \mathcal{A})$ in $T O P$ is given by $i d_{X}:\left(X, \mathcal{A}_{\tau_{\mathcal{A}}}\right) \rightarrow(X, \mathcal{A})$ where $\mathcal{T}_{\mathcal{A}}$ is the topology on $X$ determined by the neighborhood system

$$
\mathcal{N}_{\mathcal{A}}(x):=\{\{\varphi<\varepsilon\} \mid \varphi \in \mathcal{A}(x), \varepsilon \in] 0, \infty[\}, \quad \forall x \in X
$$

or equivalently by the closure operator $\bar{A}:=\{x \in X \mid \delta(x, A)=0\}$ for every $A \in 2^{X}$.

The construct $p q-M E T^{\infty}$ of extended $p q$-metric spaces and non-expansive maps too can be embedded as a bicoreflective subconstruct of $A P$. The embedding is given by $(X, d) \rightarrow\left(X, \mathcal{A}_{d}\right)$ leaving morphisms unaltered and where $\mathcal{A}_{d}$ is the approach system

$$
\mathcal{A}_{d}(x):=\left\{\varphi \in[0, \infty]^{X} \mid \varphi \leq d(x, \cdot)\right\}, \quad \forall x \in X
$$

with obvious basis consisting of the single element $d(x, \cdot)$. As to be expected the associated distance is given by $\delta(x, A)=\inf _{a \in A} d(x, a)$.

As for topological spaces a convergence theory can be developed in $A P$ (see $\mathrm{E}$. and $\mathrm{R}$. Lowen $[5,6]$ for more details). The difference with topological spaces however is that with each filter and each point we can give a distance the point "is away from being a limit point" of the filter. Precisely this goes as follows. Given a set $X, F(X)$ is the set of all filters on $X$; if $\mathcal{F} \in F(X)$, then $U(\mathcal{F})$ is the set of all ultrafilters finer than $\mathcal{F}$. If $\mathcal{G} \subset 2^{X}$ then stackx $\mathcal{G}:=\{B \subset X \mid \exists G \in \mathcal{G}: G \subset B\}$, 
if $\mathcal{G}$ consists of a single set $G$ we write $\operatorname{stack}_{X} G$ and if moreover $G$ consists of a single point $a$, we write stack $x_{X} a$ for short. If no confusion can occur, we drop the subscript $X$. Also, if $F \subset X$ we abbreviate $U(\operatorname{stack} F)$ by $U(F)$. The set $\sec \mathcal{F}$ is defined as the union of all ultrafilters finer than $\mathcal{F}$, which means sec $\mathcal{F}:=\{A \subset X \mid \forall F \in \mathcal{F}: A \cap F \neq \emptyset\}$. Let $(X, \delta)$ be an approach space and $\mathcal{F} \in F(X)$, then the limit (-function) of $\mathcal{F}$ is defined as

$$
\lambda_{\delta} \mathcal{F}(x):=\sup _{A \in \sec \mathcal{F}} \delta(x, A), \quad \forall x \in X
$$

It will be useful also to have a description of $\lambda_{\delta}$ not in terms of $\delta$ but in terms of the associated approach system $\mathcal{A}$.

PROPOSITION 2.3 [7] Let $(X, \mathcal{A})$ be an approach space. For any $\mathcal{F} \in F(X)$ and $x \in X$ we have

$$
\lambda_{\mathcal{A}} \mathcal{F}(x)=\sup _{\varphi \in \mathcal{A}(x)} \inf _{F \in \mathcal{F}} \sup _{y \in F} \varphi(y)
$$

where both $\mathcal{A}$ and $\mathcal{F}$ may be replaced by bases.

It is worthwile to mention that limits also as in TOP provide yet a third way to describe approach spaces (see E. and R. Lowen [5] for more details). For us it suffices to mention that the distance $\delta$ can be recovered from $\lambda_{\delta}$ by

$$
\delta(x, A)=\inf _{\mathcal{U} \in U(A)} \lambda_{\delta} \mathcal{U}(x)
$$

Convergence in the topological bicoreflection $\left(X, \mathcal{T}_{\delta}\right)$ of an approach space $(X, \delta)$ can easily be derived from the limit associated with $\delta$. If $\mathcal{F} \in F(X)$ then $\mathcal{F} \rightarrow x$ in $\mathcal{T}_{\delta}$ if and only if $\lambda_{\delta} \mathcal{F}(x)=0$.

In the case of $\infty$-pq-metric spaces the associated limit takes on a more simple and intuitive form. If $\mathcal{F} \in F(X)$ then

$$
\lambda_{d} \mathcal{F}(x)=\inf _{F \in \mathcal{F}} \sup _{y \in F} d(x, y),
$$

and in case $\mathcal{F}$ is generated by a sequence $\left(x_{n}\right)_{n}$ this simply means that $\lambda_{d} \mathcal{F}(x)=\lim \sup d\left(x, x_{n}\right)$.

DEFINITION 2.4 Given an approach space $(X, \mathcal{A})$, we define the measure of compactness of $X$ as

$$
\mu_{c}(X):=\sup _{\mathcal{U} \in U(X)} \inf _{x \in X} \lambda \mathcal{U}(x)
$$

The idea behind this definition is the following. Compactness means every ultrafilter should have a convergence point. Therefore the information given by $\mu_{c}$ is based on the verification for all ultrafilters what are their "best convergence" points. Before all else we give a number of equivalent forms of this definition.

PROPOSITION 2.5 [2] For any approach space $(X, \mathcal{A})$, we have

$$
\begin{aligned}
\mu_{c}(X) & =\sup _{\varphi \in \prod_{x \in X} \mathcal{A}(x)} \inf _{Y \in 2^{(X)}} \sup _{z \in X^{\prime} \inf _{x \in Y} \varphi(x)(z)} \\
& =\sup _{\psi \in \prod_{x \in X^{\Lambda(x)}}} \inf _{Y \in 2^{(X)}} \sup _{z \in X} \inf _{x \in Y} \psi(x)(z),
\end{aligned}
$$

where $\Lambda$ is a basis for the approach system $\mathcal{A}$. 
THEOREM 2.6 [2]

1. If $\left(X, \mathcal{A}_{\mathcal{T}}\right)$ is a topological approach space then $(X, \mathcal{T})$ is compact if and only if $\mu_{c}(X)=0$.

2. If $\left(X, \mathcal{A}_{d}\right)$ is an $\infty$-pq-metric approach space then $(X, d)$ is totally bounded if and only if $\mu_{c}(X)=0$.

3. If $\left(X, \mathcal{A}_{d}\right)$ is a pq-metric approach space then $(X, d)$ is bounded if and only if $\mu_{c}(X)<\infty$.

THEOREM 2.7 [2]

1. If $(X, \mathcal{A})$ and $\left(X^{\prime}, \mathcal{A}^{\prime}\right)$ are approach spaces and $f:(X, \mathcal{A}) \longrightarrow\left(X^{\prime}, \mathcal{A}^{\prime}\right)$ is a surjective contraction then $\mu_{c}\left(X^{\prime}\right) \leq \mu_{c}(X)$.

2. If $\left(X, \mathcal{A}_{j}\right)_{j \in J}$ is a family of approach spaces then

$$
\mu_{c}\left(\prod_{j \in J} X_{j}\right)=\sup _{j \in J} \mu_{c}\left(X_{j}\right) .
$$

\section{SOME NOTIONS OF LOCAL COMPACTNESS IN AP}

In this section we will define some notions of local compactness and basis-local compactness in $A P$, which on topological spaces coincide with the topological notions of local compactness and basis-local compactness. We will denote the notions of local compactness by LC $n$ where $n$ is a number between 1 and 5 , and the associated notions of basis-local compactness likewise by BLC $n$.

DEFINITION 3.1 Let $(X, \mathcal{A})$ be an approach space.

1. $(X, \mathcal{A})$ is LC1 if and only if its topological coreflection is locally compact.

2. Define

$$
\begin{aligned}
(X, \mathcal{A}) \text { is LC2 } & \Longleftrightarrow \forall x \in X, \forall \mathcal{F} \rightarrow x, \exists F \in \mathcal{F}: \mu_{c}(F)=0 \\
& \Longleftrightarrow \forall x \in X, \exists V \in \mathcal{V}(x): \mu_{c}(V)=0,
\end{aligned}
$$

where $\mathcal{V}(x)$ is the neighborhood filter of $x$ in the topological coreflection.

3. Define

$$
\begin{aligned}
(X, \mathcal{A}) \text { is } L C 3 \Longrightarrow & \forall x \in X, \forall \mathcal{F} \rightarrow x: \inf _{F \in \mathcal{F}} \mu_{c}(F)=0 \\
\Longleftrightarrow & \forall x \in X: \inf _{V \in \mathcal{V}(x)} \mu_{c}(V)=0 \\
\Longleftrightarrow & \forall x \in X, \forall \varepsilon>0, \exists \varphi \in \mathcal{A}(x), \exists \delta>0: \\
& \mu_{c}(\{\varphi<\delta\}) \leq \varepsilon .
\end{aligned}
$$

4. $(X, \mathcal{A})$ is LC4 if and only if

$$
\forall x \in X, \forall \varepsilon>0: \inf _{F \in \mathcal{V}_{\varepsilon}(x)} \mu_{c}(F) \leq \varepsilon
$$

where $\mathcal{V}_{\varepsilon}(x):=\operatorname{stack}\{\{\varphi<\varepsilon\} \mid \varphi \in \mathcal{A}(x)\}$ 
5. $(X, \mathcal{A})$ is LC5 if and only if

$$
\forall x \in X, \forall \varepsilon>0: \inf _{F \in \mathcal{V}_{\varepsilon}(x)} \mu_{c}(F) \leq \inf _{y \in X} \lambda \mathcal{V}_{\varepsilon}(x)(y)
$$

It is easily verified that the given definitions of LC2 are equivalent, and likewise are those of LC3. We have the following obvious relations between the different $\mathrm{LC} n$.

PROPOSITION $3.2 L C 1 \Longrightarrow L C 2 \Longrightarrow L C 3 \Longleftarrow L C 4 \Longleftarrow L C 5$.

PROPOSITION 3.3 Let $(X, \mathcal{T})$ be a topological space.

Then $(X, \mathcal{T})$ is locally compact $\Leftrightarrow\left(X, \mathcal{A}_{\mathcal{T}}\right)$ is LC1 $\Leftrightarrow\left(X, \mathcal{A}_{\mathcal{T}}\right)$ is LC2 $\Leftrightarrow\left(X, \mathcal{A}_{\mathcal{T}}\right)$ is LC3 $\Leftrightarrow$ $\left(X, \mathcal{A}_{\mathcal{T}}\right)$ is $L C 4 \Leftrightarrow\left(X, \mathcal{A}_{\mathcal{T}}\right)$ is $L C 5$.

This is a straightforward result and it shows the LCn can be considered as generalizations of topological local compactness in the context of approach spaces. Notice that for a topological space, $\mathcal{V}_{\varepsilon}(x)=\mathcal{V}(x)$ for every $\varepsilon>0$ and every $x \in X$.

PROPOSITION 3.4 1. Let $(X, d)$ be an $\infty$-pq-metric space. Then $(X, d)$ is LC2 if and only if every point possesses a totally bounded neighborhood.

2. Every $\infty$-pq-metric space is $L C 3$ and $L C 4$.

\section{PROOF.}

1. Let $(X, d)$ be an $\infty$-pq-metric space. Then $(X, d)$ is LC2 if and only if $\forall x \in X, \exists V \in \mathcal{V}(x)$ : $\mu_{c}(V)=0$. Denote the open ball with center $y$ and radius $\varepsilon$ by $B(y, \varepsilon)$, then $\mu_{c}(V)=0$ if and only if $\forall \varepsilon>0, \exists Y \in 2^{(V)}: V \subset \bigcup_{y \in Y} B(y, \varepsilon)$, if and only if $V$ is totally bounded.

2. Since LC4 implies LC3 we only have to show every $\infty$-pq-metric space $(X, d)$ is LC4. Now $(X, d)$ is LC4 if and only if for every $x \in X$ and every $\varepsilon>0, \inf _{F \in \mathcal{V}_{e}(x)} \mu_{c}(F) \leq \varepsilon$. But $B(x, \varepsilon)$ belongs to $\mathcal{V}_{\varepsilon}(x)$ and by adapting the first part of the proof we see $\mu_{c}(B(x, \varepsilon)) \leq \varepsilon$.

For every $\mathrm{LC} n$, we will introduce a corresponding notion $\mathrm{BLC} n$ of basis-local compactness.

DEFINITION 3.5 Let $(X, \mathcal{A})$ be an approach space.

1. $(X, \mathcal{A})$ is BLC1 if and only if its TOP-coreflection is basis-locally compact.

2. $(X, \mathcal{A})$ is BLC2 if and only if

$$
\forall x \in X, \forall V \in \mathcal{V}(x), \exists W \in \mathcal{V}(x): W \subset V \text { and } \mu_{c}(W)=0
$$

3. $(X, \mathcal{A})$ is $B L C 3$ if and only if

$$
\forall x \in X: \sup _{V \in \mathcal{V}(x)} \inf _{\substack{W \in \mathcal{V}(x) \\ W \subset V}} \mu_{c}(W)=0 .
$$

4. $(X, \mathcal{A})$ is $B L C 4$ if and only if

$$
\forall x \in X, \forall \varepsilon>0, \forall F \in \mathcal{V}_{\varepsilon}(x), \forall \varepsilon^{\prime}<\varepsilon: \inf _{\substack{G \in \mathcal{V}_{\varepsilon^{\prime}}^{\prime}(x) \\ G \subset \mathcal{C} F}} \mu_{c}(G) \leq \varepsilon^{\prime}
$$


5. $(X, \mathcal{A})$ is BLC5 if and only if

$$
\forall x \in X, \forall \varepsilon>0, \forall F \in \mathcal{V}_{\varepsilon}(x), \forall \varepsilon^{\prime}<\varepsilon: \inf _{\substack{G \in \mathcal{V}^{\prime}(x) \\ G \mathcal{C}^{\prime} F}} \mu_{c}(G) \leq \inf _{y \in X} \lambda \mathcal{V}_{\varepsilon^{\prime}}(x)(y)
$$

If an approach space is BLCn, then it is also LCn. In order to see this, notice that we have $\mathcal{V}_{\varepsilon}(x)=\cap_{\varepsilon^{\prime}<\varepsilon} \mathcal{V}_{\varepsilon^{\prime}}(x)$ for every $x \in X$ and $\varepsilon>0$. The relations between the different BLCn are as for the $\mathrm{LC} n$ :

PROPOSITION 3.6 BLC1 $\Longrightarrow B L C 2 \Longrightarrow B L C 3 \Longleftarrow B L C 4 \Longleftarrow B L C 5$.

PROPOSITION 3.7 Let $(X, \mathcal{T})$ be a topological space.

Then $(X, \mathcal{T})$ is basis-locally compact $\Leftrightarrow\left(X, \mathcal{A}_{\mathcal{T}}\right)$ is BLC1 $\Leftrightarrow\left(X, \mathcal{A}_{\mathcal{T}}\right)$ is BLC2 $\Leftrightarrow\left(X, \mathcal{A}_{\mathcal{T}}\right)$ is BLC3 $\Leftrightarrow\left(X, \mathcal{A}_{\tau}\right)$ is $B L C 4 \Leftrightarrow\left(X, \mathcal{A}_{\tau}\right)$ is BLC5.

This result illustrates the BLCn can be viewed as generalizations of topological basis-local compactness in the context of approach spaces. For $\infty$-pq-metric spaces, we get the following result.

PROPOSITION 3.8 1 . Let $(X, d)$ be an $\infty$-pq-metric space. Then $(X, d)$ is BLC2 if and only if every point possesses a neighborhood basis consisting of totally bounded neighborhoods.

2. Every $\infty$-pq-metric space is BLC3 and BLC4.

For some notions of local compactness in $A P$ we can introduce a measure of local compactness which is a generalization in which all the nice properties are preserved.

DEFINITION 3.9 Let $(X, \mathcal{A})$ be an approach space.

$$
\begin{aligned}
& \mu_{L C 3}(X):=\sup _{x \in X} \inf _{V \in \mathcal{V}(x)} \mu_{c}(V) . \\
& \mu_{B L C 3}(X):=\sup _{x \in X} \sup _{V \in \mathcal{V}(x)} \inf _{\substack{W \in \mathcal{V}(x) \\
W \subset V}} \mu_{c}(W) . \\
& \mu_{L C 4}(X):=\sup _{x \in X} \sup _{\varepsilon>0}\left(\inf _{F \in \mathcal{V}_{\varepsilon}(x)} \mu_{c}(F)-\varepsilon\right) \vee 0 . \\
& \mu_{B L C 4}(X):=\sup _{x \in X} \sup _{\varepsilon>0} \sup _{F \in \mathcal{V}_{\varepsilon}(x)} \sup _{\varepsilon^{\prime}<\varepsilon}\left(\inf _{\substack{G \in \mathcal{V}^{\prime} \\
G \subset F}} \mu_{c}(G)-\varepsilon^{\prime}\right) \vee 0 . \\
& \mu_{L C 5}(X):=\sup _{x \in X} \sup _{\varepsilon>0}\left(\inf _{F \in \mathcal{V}_{\varepsilon}(x)} \mu_{c}(F)-\inf _{y \in X} \lambda \mathcal{V}_{\varepsilon}(x)(y)\right) \vee 0 . \\
& \mu_{B L C 5}(X):=\sup _{x \in X} \sup _{\varepsilon>0} \sup _{F \in \mathcal{V}_{\varepsilon}(x)} \sup _{\varepsilon^{\prime}<\varepsilon}\left(\inf _{\substack{G \in \mathcal{V}^{\prime}(x) \\
G C^{\prime} F}} \mu_{c}(G)-\inf _{y \in X} \lambda \mathcal{V}_{\varepsilon^{\prime}}(x)(y)\right) \vee 0 .
\end{aligned}
$$

For $n \in\{3,4,5\}, \mu_{(B) L C n}(X)$ is called the measure of (basis-) local compactness of $X$. This is justified by the following proposition.

PROPOSITION 3.10 If $(X, \mathcal{A})$ is an approach space and $n \in\{3,4,5\}$, then $\mu_{(B) L C n}(X)=0$ if and only if $(X, \mathcal{A})$ is $(B) L C n$.

The following inequalities can easily be checked.

PROPOSITION 3.11 Let $(X, \mathcal{A})$ be an approach space. Then

1. For all $n \in\{3,4,5\}: \mu_{L C n}(X) \leq \mu_{B L C n}(X)$.

2. $\mu_{(B) L C 3}(X) \leq \mu_{(B) L C 4}(X) \leq \mu_{(B) L C 5}(X)$. 


\section{COUNTEREXAMPLES}

Proposition 3.2 gives some relations between the different notions of local compactness in AP. In this section, we will show these relations are really all there is to find, except perhaps LC4 $\Rightarrow$ LC5.

1. Note that there exist approach spaces which are not LC3, for instance, every topological space which does not possess local compactness.

2. To prove $\mathrm{LC} 2 \nRightarrow \mathrm{LC} 1$, just consider the metric space $(Q, d)$ of rational numbers with the euclidean metric. Every point possesses a totally bounded neighborhood, so $(Q, d)$ is LC2. But clearly the topological coreflection is not locally compact, so $(\mathscr{Q}, d)$ is not LC1.

3. We shall now show LC5 $\nRightarrow$ LC2. To this end, consider the metric (Hilbert) space $\left(l^{2}, d\right)$. Since it is a metric space, $\left(l^{2}, d\right)$ is LC4. We already know that for all $x \in l^{2}$ and all $\varepsilon>0$, $\inf _{y \in l^{2}} \lambda \mathcal{V}_{\varepsilon}(x)(y) \leq \varepsilon$. We shall prove that also $\inf _{y \in l^{2}} \lambda \mathcal{V}_{\varepsilon}(x)(y) \geq \varepsilon$ :

$$
\begin{aligned}
\inf _{y \in l^{2}} \lambda \mathcal{V}_{\varepsilon}(x)(y) & =\inf _{y \in l^{2}} \sup _{A \in \sec \mathcal{V}_{\varepsilon}(x)} \delta(y, A) \\
& =\inf _{y \in l^{2}} \sup _{A \cap B(x, \varepsilon) \neq \emptyset} \inf _{a \in A} d(y, a) .
\end{aligned}
$$

Now take $y \in l^{2}$. If $y \notin B(x, \varepsilon)$, define $A:=\{x\}$, then it is obvious that $A \cap B(x, \varepsilon) \neq \emptyset$ and $\inf _{a \in A} d(y, a)=d(y, x) \geq \varepsilon$. If $y \in B(x, \varepsilon) \backslash\{x\}$, we can find some $y^{\prime} \in B(x, \varepsilon)$ such that $d\left(y, y^{\prime}\right) \geq \varepsilon$. In that case, choose $A:=\left\{y^{\prime}\right\}$. If $y=x$ then $\sup _{A \cap B(x, \varepsilon) \neq \emptyset} \inf _{a \in A} d(x, a)=\varepsilon$. Hence we can conclude inf $y \in l^{2} \lambda \mathcal{V}_{\varepsilon}(x)(y)=\varepsilon$. This implies $\left(l^{2}, d\right)$ is also LC5. Since no point in $\left(l^{2}, d\right)$ has a totally bounded neighborhood, $\left(l^{2}, d\right)$ is not LC2.

4. In order to see $\mathrm{LC} 1 \nRightarrow \mathrm{LC} 4$, consider the following approach space $(X, \lambda)$ : Let $X$ be a set, $\mathcal{F}$ a filter on $X$ and $f: X \rightarrow[0, \infty]$ a function. If $\mathcal{G}$ is a filter on $X$ and $x \in X$, we define

$$
\lambda \mathcal{G}(x)=\lambda_{(\mathcal{F}, f)} \mathcal{G}(x):= \begin{cases}f(x) & \text { if } \mathcal{F} \cap \operatorname{stack} x \subset \mathcal{G} \text { and } \mathcal{G} \neq \operatorname{stack} x \\ \infty & \text { if } \mathcal{F} \cap \operatorname{stack} x \not \subset \mathcal{G} \\ 0 & \text { if } \mathcal{G}=\operatorname{stack} x\end{cases}
$$

In $\mathrm{E}$. and $\mathrm{R}$. Lowen [6] it was shown that $(X, \lambda)$ is an approach space. Moreover, if $\mathcal{U}$ is an ultrafilter on $X$ and $x \in X$,

$$
\lambda \mathcal{U}(x)= \begin{cases}f(x) & \text { if } \mathcal{F} \subset \mathcal{U} \text { and } \mathcal{U} \neq \operatorname{stack} x \\ \infty & \text { if } \mathcal{F} \not \subset \mathcal{U} \text { and } \mathcal{U} \neq \operatorname{stack} x \\ 0 & \text { if } \mathcal{U}=\operatorname{stack} x\end{cases}
$$

If there exists an $x \in X$ such that $\mathcal{U}=\operatorname{stack} x$, then $\inf _{x \in X} \lambda \mathcal{U}(x)=0$. If for every element $x \in X, \mathcal{U} \neq$ stack $x$ and $\mathcal{F} \subset \mathcal{U}$, then $\inf _{x \in X} \lambda \mathcal{U}(x)=\inf _{x \in X} f(x)$. If for every element $x$ of $X$, $\mathcal{U} \neq$ stack $x$ and $\mathcal{F} \not \subset \mathcal{U}$, then $\inf _{x \in X} \lambda \mathcal{U}(x)=\infty$. So we get the following three cases. If $X$ is finite (i.e., all ultrafilters on $X$ are point filters), then $\mu_{C}(X)=\sup _{\mathcal{U} \in U(X)} \inf _{x \in X} \lambda \mathcal{U}(X)=0$. If $X$ is infinite and if for every ultrafilter $\mathcal{U}$ on $X$, not being a point filter, $\mathcal{F} \subset \mathcal{U}$, then $\mu_{c}(X)=\inf _{x \in X} f(x)$. Finally, if $X$ is infinite and there exists some ultrafilter $\mathcal{U}$ on $X$, not a point filter, such that $\mathcal{F} \not \subset \mathcal{U}$, then $\mu_{c}(X)=\infty$. Let $\mathcal{B}$ be the intersection of all ultrafilters on $X$ which are not a point filter. A straightforward verification shows that $\mathcal{B}=\{X \backslash A \mid A$ is finite $\}$. Using this fact, we can state

$$
\mu_{c}(X)= \begin{cases}0 & \text { if } X \text { is finite } \\ \inf _{x \in X} f(x) & \text { if } X \text { is infinite and } \mathcal{F} \subset\{X \backslash A \mid A \text { is finite }\} \\ \infty & \text { if } X \text { is infinite and } \mathcal{F} \not \subset\{X \backslash A \mid A \text { is finite }\}\end{cases}
$$


In the sequel we will suppose $X$ is infinite, $\mathcal{F}$ is an ultrafilter on $X$ with only infinite elements, and $f \not \equiv \infty$. Then for every ultrafilter $\mathcal{U}$ on $X$,

$$
\lambda \mathcal{U}(x)= \begin{cases}f(x) & \text { if } \mathcal{F}=\mathcal{U} \\ \infty & \text { if } \mathcal{F} \neq \mathcal{U} \text { and } \mathcal{U} \neq \operatorname{stack} x \\ 0 & \text { if } \mathcal{U}=\operatorname{stack} x\end{cases}
$$

In order to know $\mathcal{V}_{\varepsilon}(x)$ we need to find $\mathcal{A}(x)$.

$$
\begin{aligned}
\varphi \in \mathcal{A}(x) & \Leftrightarrow \forall \mathcal{U} \in U(X): \sup _{U \in \mathcal{U}} \inf _{z \in U} \varphi(z) \leq \lambda \mathcal{U}(x) \\
& \Leftrightarrow \sup _{F \in \mathcal{F}} \inf _{z \in F} \varphi(z) \leq f(x) \text { and } \sup _{U \in \operatorname{stack} x} \inf _{z \in U} \varphi(z)=0 \\
& \Leftrightarrow \sup _{F \in \mathcal{F}} \inf _{z \in F} \varphi(z) \leq f(x) \text { and } \varphi(x)=0 .
\end{aligned}
$$

We infer

$$
\mathcal{V}_{\varepsilon}(x)=\operatorname{stack}\left\{\{\varphi<\varepsilon\} \mid \sup _{F \in \mathcal{F}} \inf _{z \in F} \varphi(z) \leq f(x) \text { and } \varphi(x)=0\right\} .
$$

Notice that for any $G \subset X$ the initial structure on $G$ is given by $\lambda_{\left(\mathcal{F}_{\mid G}, f_{\mid}\right)}$and consequently the expression for $\mu_{c}(G)$ is obtained by replacing $X$ by $G$ in the expression for $\mu_{c}(X)$. Hence we get

$$
\begin{aligned}
& \inf _{G \in \mathcal{V}_{\varepsilon}(x)} \mu_{c}(G)=0 \text { if there exists some finite } G \in \mathcal{V}_{\varepsilon}(x) \\
& \inf _{G \in \mathcal{V}_{\varepsilon}(x)} \mu_{c}(G)=\inf _{\left\{G \backslash A \mid A \in \mathcal{V}_{\varepsilon}(x) \text { is finite }\right\} \supset \mathcal{F}_{\mid G}} \inf _{z \in G} f(z) \text { if every } G \in \mathcal{V}_{\varepsilon}(x) \\
& \text { is infinite and there exists some } G \in \mathcal{V}_{\varepsilon}(x) \text { such that } \\
& \{G \backslash A \mid A \text { is finite }\} \supset \mathcal{F}_{\mid G} \text {, and } \\
& \inf _{G \in \mathcal{V}_{\varepsilon}(x)} \mu_{c}(G)=\infty \text { if every } G \in \mathcal{V}_{\varepsilon}(x) \text { is infinite and satisfies } \\
& \{G \backslash A \mid A \text { is finite }\} \not \supset \mathcal{F}_{\mid G} \text {. }
\end{aligned}
$$

Consider some $x \in X$ such that $f(x)<\infty$. If we take $\varepsilon>f(x)$ then $\mathcal{V}_{\varepsilon}(x)$ contains only infinite elements: if $\varphi$ satisfies $\sup _{F \in \mathcal{F}} \inf _{z \in F} \varphi(z) \leq f(x)$ and $\varphi(x)=0$, then for every $F \in \mathcal{F}$ and for every $\theta>0$, there exists some $z \in F$ such that $\varphi(z) \leq f(x)+\theta$, so $\{\varphi<\varepsilon\}$ is infinite, for $\mathcal{F}$ is an ultrafilter containing only infinite elements. The fact that $\mathcal{F}$ is an ultrafilter also implies that $\{G \backslash A \mid A$ is finite $\} \not \supset \mathcal{F}_{\mid G}$ for every $G \in \mathcal{V}_{\varepsilon}(x)$, So we can conclude $\inf _{G \in \mathcal{V}_{\varepsilon}(x)} \mu_{c}(G)=\infty>\varepsilon$, whence $(X, \lambda)$ is not LC4.

Now choose a function $f$ as before, but moreover satisfying $f>0$. For every $x \in X$, $\mathcal{V}(x)=\operatorname{stack} x$, so $\{x\}$ is a compact neighborhood of $x$. This means $(X, \lambda)$ is LC1.

\section{REFERENCES}

[1] LOWEN, E., LOWEN, R., and VERBEECK, C., Exponential objects in the construct PRAP, UIA internal report 96-09 (1996).

[2] LOWEN, R., Kuratowski's measure of non-compactness revisited, Quart. J. Math. Oxford (2) 39 (1988), 235-254.

[3] LOWEN, R., Approach spaces: the missing link in the topology-uniformity-metric triad, Oxford Mathematical Monographs, Oxford University Press (1997). 
[4] ADÁMEK, J., HERRLICH, H., and STRECKER, G.E., Abstract and concrete categories, John Wiley (1990).

[5] LOWEN, E. and LOWEN, R., A quasitopos containing CONV and MET as full subcategories, Internat. J. Math. \& Math. Sci. 3 (1988), 417-438.

[6] LOWEN, E. and LOWEN, R., Topological quasitopos hulls of categories containing topological and metric objects, Cah. Top. Géo. Diff. Cat. 30:3 (1989), 213-228.

[7] LOWEN, R., A topological category suited for approximation theory?, J. Approximation Theory 56 (1989), 108-117. 


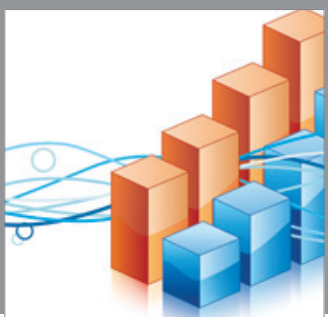

Advances in

Operations Research

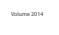

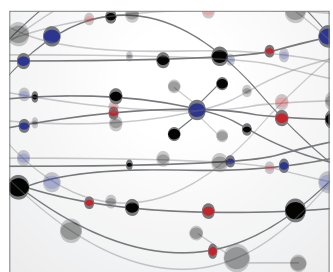

\section{The Scientific} World Journal
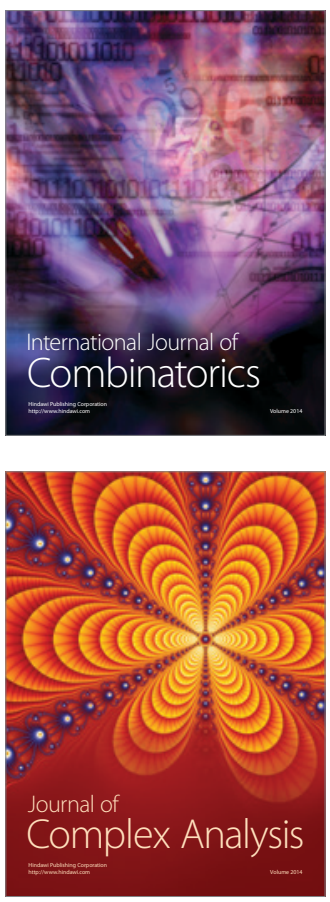

International Journal of

Mathematics and

Mathematical

Sciences
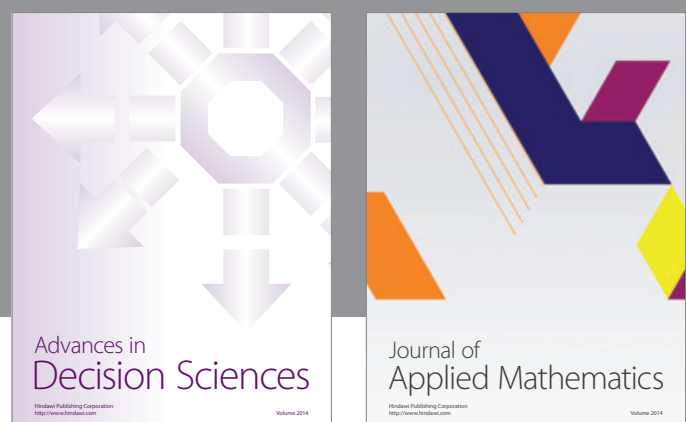

Journal of

Applied Mathematics
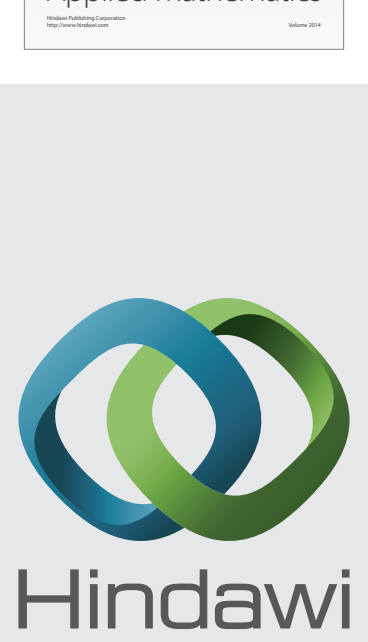

Submit your manuscripts at http://www.hindawi.com
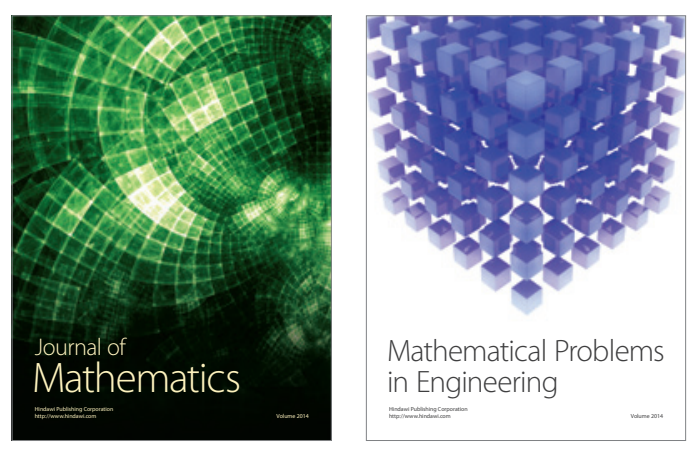

Mathematical Problems in Engineering
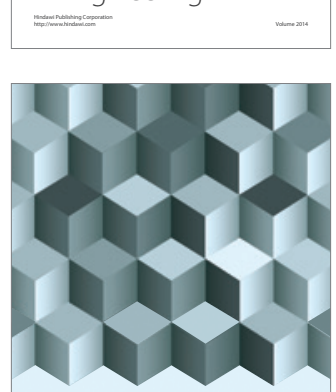

Journal of

Function Spaces
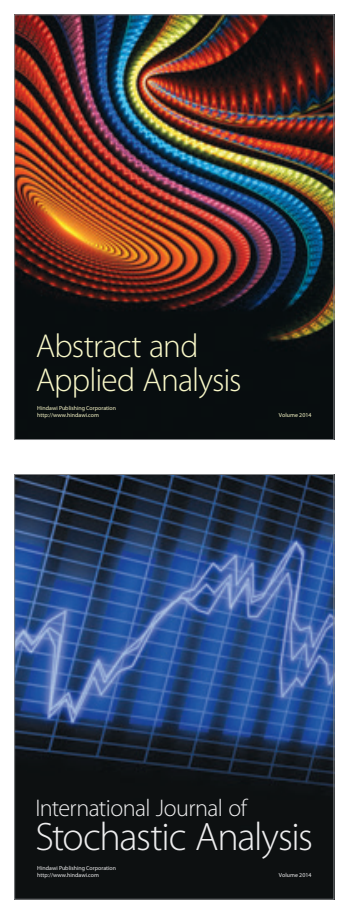

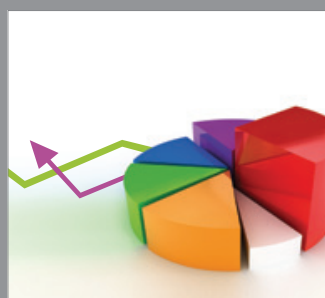

ournal of

Probability and Statistics

Promensencen
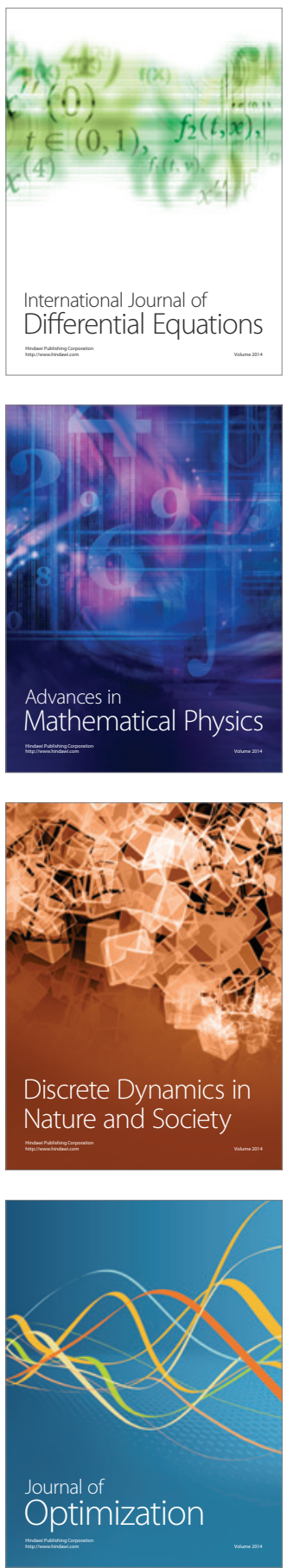